\section{Familial \\ retinoblastoma: fundus screening schedule impact and guideline proposal. A retrospective study}

P-R Rothschild' ${ }^{1}$, D Lévy², A Savignoni ${ }^{3}$, L Lumbroso-Le Rouic' ${ }^{1}$, I Aerts², M Gauthier-Villars', M Esteve ${ }^{5}$, D Bours ${ }^{3}$, L Desjardins ${ }^{1}$, F Doz ${ }^{2}$ and C Lévy-Gabriel'

\section{Abstract}

Aims To assess if systematic fundus screening according to an 'intensive' schedule alters ocular outcome and to propose fundus screening schedule guidelines for children related to a retinoblastoma patient.

Methods For children with a positive family history of retinoblastoma, we perform fundus exams shortly after birth under general anaesthesia and then at regular intervals according to schedules based on the risk. Familial retinoblastoma cases seen at our institution from January 1995 to December 2004 were retrospectively classified as 'screened' or 'non-screened' (NS) and, among the 'screened' patients, as 'intensively screened' (IS) if screening matched our recommendations or 'non-intensively screened' (S). Groups were compared by Fisher exact test for categorical variables and Kruskal-Wallis test for continuous variables. Results Among the 547 retinoblastoma patients managed at our institution during this period, 59 were familial cases. In all, 20 were in the NS group, 23 in the $S$ group, and 16 in the IS group. The number of children enucleated was, respectively, 13,2 , and 0 $\left(P<10^{-4}\right)$; external beam radiation (EBRT) was required for, respectively, 6,0 , and 2 children $(P<0.009)$. Chemotherapy burden and visual acuity were not significantly different between groups.

Conclusion An 'intensive' fundus screening schedule decreased the need for enucleation and EBRT. Therefore, despite the heavy burden of the screening schedule, we recommend physicians and health-care professionals to better inform and refer children with a family history of retinoblastoma for genetic counselling and proper fundus screening in specialized centres.

Eye (2011) 25, 1555-1561; doi:10.1038/eye.2011.198; published online 16 September 2011

Keywords: retinoblastoma; cancer; familial; hereditary; screening; outcome

\section{Introduction}

Retinoblastoma (RB) is the most frequent malignant intraocular tumour in children with an incidence of 1 case per 17000 births. ${ }^{1}$ Most cases are sporadic and only $10 \%$ are familial (defined by a positive family history of RB). All patients having presented a bilateral $\mathrm{RB}$ and up to $10 \%$ having presented a unilateral $\mathrm{RB}$ are expected to carry a germinal mutation and can thus transmit the disease to their offspring. At-risk children should be screened very early in life by regular fundus examinations in order to diagnose the tumour in its initial stages. It has been shown that early diagnosis is associated with less advanced disease and better outcome in terms of globe salvage. ${ }^{2,3}$

In this respect early detection seems critical, but to date no agreement exists on the best screening schedule to adopt and the evidence on the topic is scarce. Only few studies have shown interesting results in terms of ocular conservation and final visual acuity (VA) in patients with positive family history screened early-in-life by fundus examinations at regular intervals and under general anaesthesia. ${ }^{2,4-6}$ Nevertheless, these were small uncontrolled studies with poorly defined screening schedules and included patients treated before the advent
${ }^{1}$ Department of Ocular Oncology, Institut Curie, Paris, France

${ }^{2}$ Department of Paediatric Oncology, Institut Curie, Paris, France

${ }^{3}$ Department of Biostatistics, Institut Curie, Paris, France

${ }^{4}$ Department of Genetic Oncology, Institut Curie, Paris, France

${ }^{5}$ Department of Anaesthesiology, Institut

Curie, Paris, France

Correspondence: L Lumbroso-Le Rouic, Department of Ocular Oncology, Institut Curie, 26 rue d'Ulm 75005 Paris, France

Tel + 33144324 603; Fax + 33153104018. E-mail: livia.lumbroso@ curie.net

Received: 11 August 2010 Accepted in revised form: 12 July 2011

Published online:

16 September 2011

Meeting presentation: Ocular Oncology Group Meeting, Jerusalem, April 2009 (oral presentation): Congrès de la Société Française d'Ophtalmologie, May 2009 (oral presentation). 
of chemotherapy as a first-line treatment for almost all familial RB patients.

To specifically address the role of an 'intensive' screening in the chemotherapy era, we compared the outcome in patients with familial retinoblastoma who were retrospectively classified as 'screened' or 'non-screened' (NS) and, among the 'screened' patients, as 'intensively screened' (IS) if screening matched our recommendations, or 'non-intensively screened' (S) if screening did not match our recommendations.

The goal of the study was to determine for the first time the specific impact of an 'intensive' screening on familial retinoblastoma in terms of enucleation, external beam radiation (EBRT) needs, chemotherapy regimen, and final VA in order to retrospectively validate our screening guidelines for children with positive family history of retinoblastoma and to possibly justify the burden for families and costs for third payers.

\section{Patients and methods}

\section{Familial retinoblastoma patients}

Since January 1995, all retinoblastoma patients were included prospectively in our database at the Curie Institute, Paris, France; the national referral centre for retinoblastoma. The presence of a positive family history was used to retrieve all familial cases of retinoblastoma defined by at least one child's relative being affected. The diagnosis of RB was essentially clinical by fundus examination using an indirect ophthalmoscope.

\section{Screening recommendations and screening groups}

Screening recommendations

Our recommendation for children with a positive family history of retinoblastoma is to perform fundus screening according to a schedule based on the absolute risk. The risk assessment depends on the clinical context and on the genetic testing results. For a child known to carry a mutation in the RB1 gene and for the child of a parent with a personal history of bilateral RB (or multifocal unilateral $\mathrm{RB}$ ) we recommend the following screening protocol: (1) fundus exam within the first 8 days after birth, then every month up to 18 months and then progressively tapered to every 3 months.

For the child of a parent with a personal history of unilateral and unifocal RB and for brothers or sisters of a bilaterally affected child (or unilateral multifocal RB) we recommend the following screening protocol: (2) fundus exam within the first month after birth, every 2 months up to 2 years of age and then every 6 months up to 4 years of age.

A summary of RB risk according to the clinical context or genetic testing and corresponding fundus screening recommendations is given in Table 1 .

The fundus examination procedure in our institution is performed on an outpatient basis except for children younger than 6 months of age who remain overnight. A short general anaesthesia without intubation is required as long as poor cooperation prevents a thorough retinal examination.

\section{Screening groups}

Patients with familial retinoblastoma, referred to the Curie Institute between January 1995 and December 2004 either for screening or at diagnosis were retrospectively classified as 'screened' or NS group and, among the 'screened' patients, as the IS group if the screening followed the above recommendations, or as the $S$ group if fundus screening did not match our recommendations.

The NS group had a fundus examination because of clinical signs, leukokoria, and strabismus being the more common presenting signs. In this group, families and health-care professionals were unaware of the necessity

Table 1 Proposed fundus screening guidelines, based on the absolute risk, depending on the clinical context or genetic testing

\begin{tabular}{|c|c|c|c|}
\hline $\begin{array}{l}\text { Clinical context or } \\
\text { genetic testing }\end{array}$ & $\begin{array}{l}\text { Risk to carry } \\
\text { germinal RB1 } \\
\text { mutation }\end{array}$ & $\begin{array}{l}\text { Overall RB risk } \\
\text { for the child }{ }^{\mathrm{a}}(\%)\end{array}$ & Fundus screening protocol recommendations \\
\hline
\end{tabular}

\begin{tabular}{|c|c|c|c|}
\hline $\begin{array}{l}\text { Children known to carry } \\
R B 1 \text { mutation }\end{array}$ & Known $(100 \%)$ & 90 & $\begin{array}{l}\text { First week after birth, then every month up to } 18 \text { months } \\
\text { of age, then every } 3 \text { months up to } 4 \text { years of age }\end{array}$ \\
\hline Parent bilaterally ${ }^{\mathrm{b}}$ affected & $100 \%$ & 45 & \\
\hline Parent unilaterally affected & $10 \%$ & 4 & $\begin{array}{l}\text { First month after birth, then every } 2 \text { months up to } 2 \text { years } \\
\text { of age, then every } 6 \text { months up to } 4 \text { years of age }\end{array}$ \\
\hline Sibling bilaterally ${ }^{\mathrm{b}}$ affected & $100 \%$ & 4 & \\
\hline
\end{tabular}

Abbreviations: RB, retinoblastoma; RB1, retinoblastoma gene.

${ }^{\text {a }}$ The risk is computed assuming a $90 \%$ penetrance of the disease and a $50 \%$ risk to inherit the mutated allele from a parent. For unaffected parents of a bilaterally affected child, the risk to carry germinal mutation for each parent is $5 \%$.

${ }^{\mathrm{b}}$ Or unilateral retinoblastoma with multifocal tumours. 
of such a screening for children with a positive family history of RB.

If at least one systematic fundus examination was performed, in the absence of clinical signs and because of the family history the child was then considered as screened.

Children screened at Curie Institute and according to the aforementioned recommendations were classified as the IS group.

For classification in the IS group, we allowed no missing fundus and a maximum delay for each programmed fundus of one week. Otherwise the child was excluded from the IS group and was reclassified into the $S$ group.

Children screened elsewhere than Curie Institute and referred to US for management after the diagnosis was made were classified in the IS group or $S$ group according to the screening performed by the referring centre.

\section{Descriptive data}

Data collected included age at diagnosis, gender, whether bilateral involvement was initially present or subsequently developed, and disease classification according to the Reese-Ellsworth Classification (RE) and the International Classification of Retinoblastoma (ICR). ${ }^{7}$ To increase the clinical relevance of the findings, we also classified each child according to his worst-affected eye.

To allow a better comparison between groups considering the change in therapeutic modalities from exclusive EBRT to chemotherapy (CT) regimens, we limited recruitment of our study population to the beginning of the CT regimens. All the patients included in the study have been treated according to the chemotherapy protocols previously described and published..$^{8-10}$ Since 1995, chemotherapy and local treatments usually represented the first-line treatment for all familial RB patients.

Treatment data included the number of children who required enucleation, EBRT, and chemotherapy. For the latter, we collected the number of children who required at least one cycle of CT and the median number of cycles per child as well as the cumulative dose of the most frequently used drugs (carboplatin and etoposide).

Monocular best-corrected VA of both eyes was evaluated but we only considered the best seeing eye of each child for statistical analysis. VA expressed in decimal fraction was assessed using a distance acuity test as soon as cooperation was sufficient.

\section{Statistical analysis}

Differences between groups were analysed by Fisher exact test for categorical variables and Kruskal-Wallis test for continuous variables. Significance level was 0.05.
Analyses were performed using ' $R$ ' version 2.5.0 software (R Foundation for Statistical Computing, Vienna, Austria; http:/ / cran.r-project.org).

\section{Ethical issues}

The database is declared according to the French legislation to the Commission Nationale Informatique et Libertés, the administrative office that authorizes and regulates the use of electronic databases. Approval from the locally appointed ethics committee was obtained.

\section{Results}

Among the 547 retinoblastoma children seen at Curie Institute from January 1995 to December 2004, 59 (11\%) had a positive family history (Figure 1). Sex ratio was close to one with 31 boys and 28 girls. Median follow-up was 8.9 years (median 107 months, range $0-161$ ) and median interval from the last evaluation was 4 months. All the patients were disease-free at last evaluation. One patient in the NS group died from a pinealoblastoma and one patient in the IS group had been successfully treated for a unilateral nephroblastoma.

All the children referred for screening purposes to our institution (Curie Institute) were referred early-in-life and were assigned to our suggested screening schedule. All were retrospectively totally compliant; therefore, none was reclassified into the $S$ group.

Conversely, all the children screened elsewhere were referred to us at the time of diagnosis when a tumour was already present. None of these children met the criteria to be classified in the IS group and were therefore classified as the $S$ group. For most of the children in the $S$ group, when the first screening fundus was performed by the referring ophthalmologist, a tumour was already present. Therefore, in the $S$ group, age at diagnosis reflects the age of the first fundus exam.

Among the 51 children (86\%) with bilateral disease, 12 children (24\%) initially presented only unilateral involvement and subsequently developed a tumour in the other eye. The median age at diagnosis was 9 months in the NS group, 4 months in the $S$ group, and 0.5 month in the IS group $\left(P<10^{-3}\right)$.

Clinical characteristics are shown in Tables 2 and 3.

As shown in Figure 2, significantly more children were present in the lower groups of both Reese-Ellsworth and ICR with 'intensive' screening $\left(P<10^{-3}\right.$ and $P<10^{-4}$ for the RE and ICR, respectively).

The numbers of children who required enucleation, EBRT therapy, and at least one cycle of chemotherapy are shown in Figure 3.

Enucleation and EBRT rates were significantly lower in screened groups (respectively, $P<10^{-4}$ and $P<0.009$ ). 


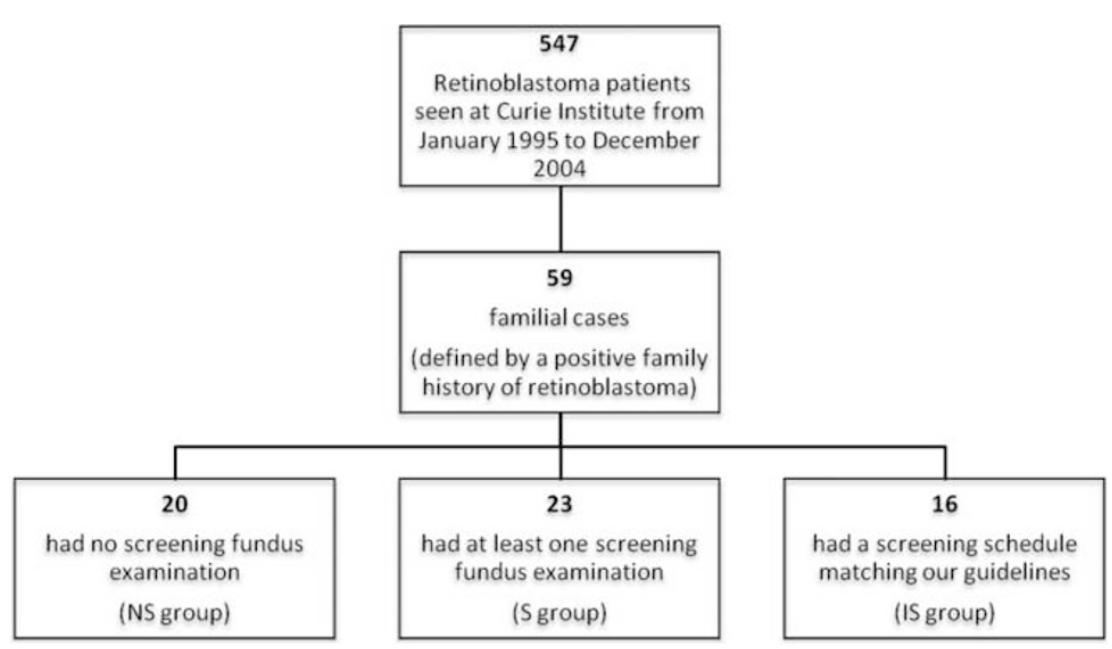

Figure 1 Groups of screening flow chart. Three screening groups were retrospectively defined according to the type of screening performed in familial cases of retinoblastoma seen at Curie Institute during the study period.

Table 2 Clinical characteristics (number of children unless otherwise specified)

\begin{tabular}{lcccc}
\hline & $\begin{array}{c}\text { Non-screened } \\
(\mathrm{n}=20)\end{array}$ & $\begin{array}{c}\text { Non-intensively } \\
\text { screened }(\mathrm{n}=23)\end{array}$ & $\begin{array}{c}\text { Intensively screening } \\
(\mathrm{n}=16)\end{array}$ & $\begin{array}{c}\text { All patients } \\
(\mathrm{n}=59)\end{array}$ \\
\hline $\begin{array}{l}\text { Unilateral RB } \\
\text { Bilateral RB }\end{array}$ & 4 & 3 & 1 & $8(14 \%)$ \\
$\begin{array}{l}\text { Median age (months) } \\
\text { at diagnosis (range) }\end{array}$ & $9(2-57)$ & 20 & 15 & $51(86 \%)$ \\
\hline
\end{tabular}

Abbreviation: RB, retinoblastoma.

Among the 15 patients requiring enucleation, 2 children in the NS group had bilateral enucleation. None of the IS children required enucleation. Eight patients underwent EBRT, all had bilateral involvement, and two of them in the NS group had both eyes irradiated.

All patients except four received at least one cycle of CT. Among those four patients, three in the NS group had enucleation as a first-line treatment for advanced unilateral disease. The fourth patient in the IS group had unilateral mild involvement amenable to laser therapy alone. The number of children requiring at least one cycle of chemotherapy was not significantly different between groups. The median number of CT cycles was, respectively, 5 for both the NS and the $S$ group and 6 for the IS group. The median cumulative doses of carboplatin and etoposide for each group are provided in Table 4 . Both the median number of CT cycles and the median cumulative carboplatin or etoposide doses were not significantly different between the three groups.

VA was available for 45 children. Distance corrected VA of the best eye was better than 0.5 for more than $60 \%$ of the children evaluated without any significant difference between the three groups. VA details are shown in Table 5.
Table 3 Reese-Ellsworth classification and international classification of retinoblastoma in number of eyes according to the screening performed

\begin{tabular}{lcccc}
\hline Classification & NS group & S group & IS group & All patients \\
\hline Reese-Ellsworth & classification & & & \\
I & 2 & 15 & 11 & 28 \\
II & 2 & 4 & 6 & 12 \\
III & 11 & 21 & 13 & 45 \\
IV & 2 & 0 & 0 & 2 \\
V & 19 & 3 & 1 & 23 \\
\multicolumn{1}{l}{ International classification of retinoblastoma } \\
A & 2 & 13 & 11 & \\
B & 8 & 23 & 18 & 26 \\
C & 2 & 5 & 1 & 89 \\
D & 11 & 1 & 0 & 12 \\
E & 8 & 1 & 0 & 9 \\
\hline
\end{tabular}

a Data available for 104 eyes.

\section{Discussion}

The data presented here tend to suggest that an 'intensive' fundus screening schedule is associated with a better outcome, especially in terms of globe sparing. 


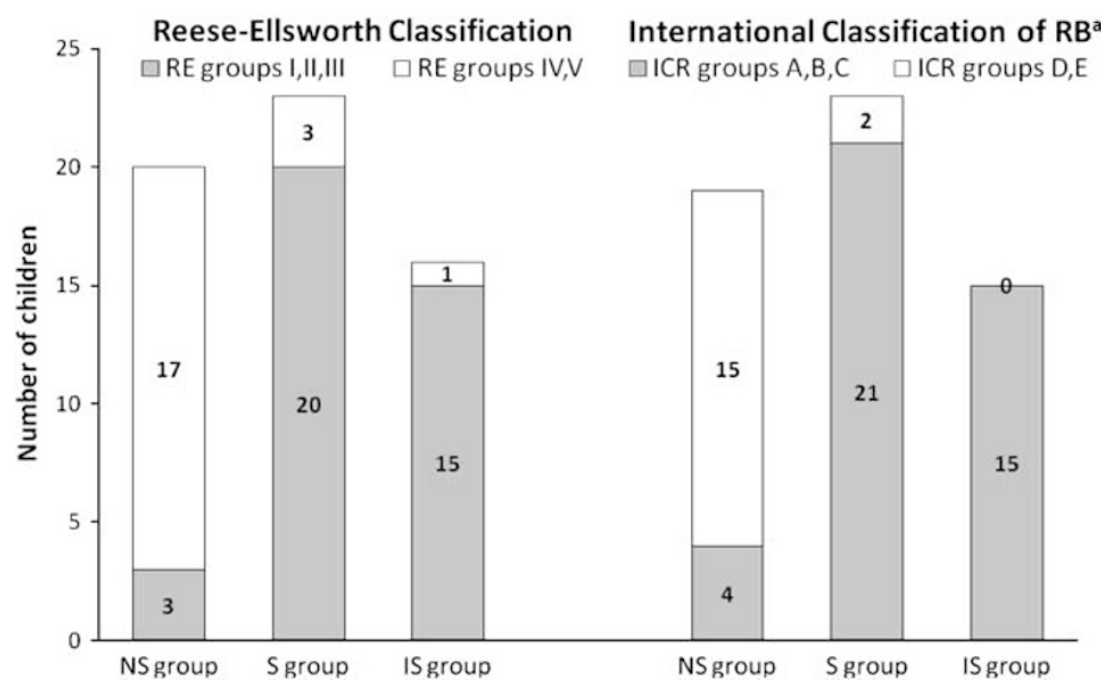

Figure 2 Disease stage at presentation according to the RE and the ICR (number of children). The worst eye of each child was considered for classification. Patients in the I, II, or III group of the RE classification (or A, B, and C group of the ICR) are usually eligible for a conservative management. Children in the IV and V group of the RE (or D, E of the ICR) require enucleation or EBRT therapy. ${ }^{6,16}$ a Data available for 57 patients.

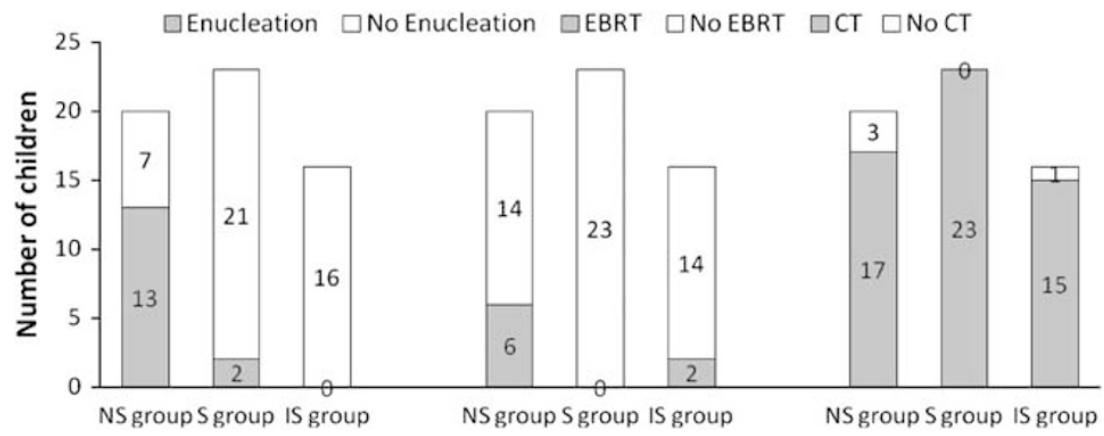

Figure 3 Management modalities according to the type of screening (number of children who received the treatment at least once). Screening significantly reduced the need for enucleation $(P<0.0001)$ and EBRT $(P<0.009)$ without significantly altering chemotherapy requirements.

Table 4 Chemotherapy cumulative doses (median) of the most used drugs, carboplatin and etoposide

\begin{tabular}{|c|c|c|c|c|c|}
\hline & $\begin{array}{l}\text { NS group } \\
(\mathrm{n}=20)\end{array}$ & $\begin{array}{l}S \text { group } \\
(\mathrm{n}=23)\end{array}$ & $\begin{array}{l}\text { IS group } \\
(\mathrm{n}=16)\end{array}$ & $\begin{array}{l}\text { All patients } \\
\quad(\mathrm{n}=59)\end{array}$ & P-value \\
\hline $\begin{array}{l}\text { Cumulative carboplatin dose (median) } \\
\text { in } \mathrm{mg} / \mathrm{m}^{2} \text { (range) }\end{array}$ & $2830(600-4880)$ & $2740(112-5040)$ & $2240(550-5600)$ & $2730(112-5600)$ & 0.98 \\
\hline $\begin{array}{l}\text { Cumulative etoposide dose (median) } \\
\text { in } \mathrm{mg} / \mathrm{m}^{2} \text { (range) }\end{array}$ & $966(450-3300)$ & $900(297-1000)$ & $900(297-1944)$ & $900(297-3300)$ & 0.58 \\
\hline
\end{tabular}

In our study, this screening decreased the enucleation and EBRT rates significantly. However, using the current screening and therapeutic approaches, early detection is not associated with a decreased use of systemic chemotherapy or a better VA.

As previously reported in the literature, family history in our study was present in $11 \%$ of RB cases. ${ }^{11}$ It is also worth noting that $14 \%$ of the children, although known to carry a germinal mutation, had only unilateral involvement. This further supports the systematic proposal for a genetic screening and genetic counselling for all RB patients including unilateral presentations.

Fundus screening, especially 'intensive' screening, according to our recommendations was significantly associated with a reduced age at diagnosis in our study. All the children screened according to our 
Table 5 Visual acuity (decimal fraction) of the best eye for each child (number of patients)

\begin{tabular}{|c|c|c|c|c|}
\hline & NS group & $S$ group & IS group & All patients $(\mathrm{n}=45)^{\mathrm{a}}$ \\
\hline \multicolumn{5}{|c|}{ Visual acuity } \\
\hline$\leqslant 0.1$ & $2(13 \%)$ & 0 & $1(7 \%)$ & $3(7 \%)$ \\
\hline$>0.1$ & $4(27 \%)$ & 0 & $3(21 \%)$ & $7(15 \%)$ \\
\hline$>0.5$ & $9(60 \%)$ & $17(100 \%)$ & $9(72 \%)$ & $35(78 \%)$ \\
\hline
\end{tabular}

${ }^{\mathrm{a}}$ Visual acuity available for 45 patients.

recommendations except one (93\%) belonged to group I, II, or III of the RE classification. In their series (only screened children) Imhof et al reported similar findings with more than $70 \%$ of their patients being diagnosed within the first 2 weeks of life and $82 \%$ being in the RE group I, II, or III. ${ }^{2,4,5,11,12}$ Although patients in the I, II, or III group of the RE classification or in the A, B, or C group of the ICR are usually amenable to an eye conservation strategy (most often systemic chemotherapy combined with focal treatments), patients with more advanced disease (group IV, V and D, E) often require aggressive treatment such as enucleation or EBRT despite chemoreduction. ${ }^{3,13}$

These results tend to suggest an association between intensive screening and early diagnosis, which probably explains the lower stages of the disease found in screened children. It is well known that early-in-life tumours tend to appear at the posterior pole close to the fovea, whereas late tumour development occurs in the far retinal periphery; this explains the large number of eyes found in the B group of the ICR classification., 72 Altogether these findings suggest that 'intensive' screening allows earlier diagnosis and therefore prevents advanced disease. However, despite early detection, macular involvement cannot be prevented.

No child screened according to our recommendations required enucleation. Imhof et al. reported a similar rate, with 2/17 screened patients enucleated (11\%).

Besides avoiding enucleation, screening also decreases the need for EBRT. Since the advent of chemotherapy in RB management and given the well-known severe complications, EBRT is only used to treat advanced disease in the only remaining eye or after a relapse, to avoid bilateral enucleation. ${ }^{14}$ This latter situation arises more frequently in the NS population as enucleation rates are significantly higher. Imhof et al reported a higher proportion of patients treated with EBRT (6/17, ie, $35 \%$ ) in their screened population as this study included patients treated from 1992, when EBRT was the reference conservative treatment. The late complications of EBRT encompass second cancers, severe cosmetic consequences including orbital bone hypoplasia, as well as functional complications, such as radiation retinopathy, cataract, or sicca syndrome. ${ }^{15}$
Chemotherapy, an alternative treatment to EBRT, dramatically changed retinoblastoma management more than a decade ago and is now the mainstay in the management of familial RB and almost all familial $\mathrm{RB}$ patients in our study received this modality as a first-line treatment. To account for this recent therapeutic change, all the children have been recruited recently on a limited period of time and all have been treated according to well-established chemotherapy protocols to ensure the comparability of the different screening groups.

Earlier and smaller tumour detection seemed not to decrease the chemotherapy needs.

Although short-term systemic side effects in our study were acceptable given the gravity of the disease and the benefit provided by $\mathrm{CT}$, the issue regarding long-term complications of CT in this population with a strong predisposition to secondary cancer has still not been addressed and demands long-term follow-up.

We found no difference between groups regarding VA results. Best-corrected monocular VA was rather good, with more than $60 \%$ of children having more than 0.5 regardless of the screening performed. Other authors also reported visual acuities better than 0.5 in more than $88 \%$ of screened children. ${ }^{4}$ Older series are not comparable, because of the frequent use of EBRT and its well-known functional complications described above compromising VA. ${ }^{16,17}$

To our knowledge, this is the first study comparing the outcome in NS children and in children screened according to different strategies. Despite the limitations owing to the retrospective nature of the study, our standardized screening schedules tailored according to the risk and according to the genetic testing along with well-established treatment protocols based on the chemoreduction paradigm ensure comparability of the groups and thus enable to isolate the specific role of screening. To prove the causality and to control for potential bias a randomized control trial would be necessary with one arm per screening protocol and one arm without screening. This latter methodology cannot be applied for obvious ethical issues. Moreover, the rarity of familial forms (approximately one case per 200000 births) and the absence of agreement regarding screening schedules or management also preclude conducting randomized control trials assessing different screening protocols. Therefore, to date we can only rely on well-conducted retrospective studies.

It is worth noting that, unfortunately, a large number of families in our study despite a positive family history of retinoblastoma were not informed about the necessity of genetic referral and the existence of screening programs. This figure, in a Western country, underscores how little ophthalmologists and other 
health-care professionals know about familial RB and how critical is education.

\section{Conclusion}

To conclude, early-in-life screening by fundus examination under general anaesthesia at regular intervals according to a schedule based on the absolute estimated risk can dramatically improve prognosis in terms of globe sparing in children with positive family history of retinoblastoma.

Therefore, patients with personal or familial history of retinoblastoma should be referred for genetic counselling. According to the risk assessment their offspring may benefit from early-in-life fundus screening in specialized centres.

\section{Summary}

\section{What was known before}

- Fundus screening in familial retinoblastoma is generally recommended and presumed to provide good ocular outcomes but to date; studies were small and uncontrolled with poorly defined screening schedules.

- What can be improved by screening and to what extent has been poorly studied because of the lack of a control group.

- Older studies used external beam radiation as a reference conservative treatment.

- No published data on the adherence of patients to screening programs in Western countries.

What this study adds

- To our knowledge, this is the first publication of a precise risk-based screening guideline. We compared large groups of non-screened versus differently screened children.

- Screening decreases enucleation and EBRT needs, but has no effect on CT burden or final VA.

- This study only includes patients treated after the advent of CT in RB management.

- Physicians and families are unaware of the necessity of specialized referral for children having a relative affected by RB.

\section{Conflict of interest}

The authors declare no conflict of interest.

\section{References}

1 Moll A, Kuik D, Bouter L, Den Otter W, Bezemer PD, Koten JW et al. Incidence and survival of retinoblastoma in The Netherlands: a register based study 1862-1995. Br J Ophthalmol 1997; 81(7): 559-562.
2 Wallach M, Balmer A, Munier F, Houghton S, Pampallona S, von der Weid $\mathrm{N}$ et al. Shorter time to diagnosis and improved stage at presentation in Swiss patients with retinoblastoma treated from 1963 to 2004. Pediatrics 2006; 118(5): e1493-e1498.

3 Shields C, Mashayekhi A, Au A, Czyz C, Leahey A, Meadows AT et al. The International Classification of Retinoblastoma predicts chemoreduction success. Ophthalmology 2006; 113(12): 2276-2280.

4 Imhof S, Moll A, Schouten-van Meeteren A. Stage of presentation and visual outcome of patients screened for familial retinoblastoma: nationwide registration in the Netherlands. Br J Ophthalmol 2006; 90(7): 875-878.

5 Abramson D, Beaverson K, Sangani P, Vora RA, Lee TC, Hochberg HM et al. Screening for retinoblastoma: presenting signs as prognosticators of patient and ocular survival. Pediatrics 2003; 112(6 Part 1): 1248-1255.

6 Chantada G, Dunkel I, Qaddoumi I, Antoneli CB, Totah A, Canturk $S$ et al. Familial retinoblastoma in developing countries. Pediatr Blood Cancer 2009; 53(3): 338-342.

7 Linn Murphree A. Intraocular retinoblastoma: the case for a new group classification. Ophthalmol Clin North Am 2005; 18(1): 41-53, viii.

8 Levy C, Doz F, Quintana E, Pacquement H, Michon J, Schlienger $\mathrm{P}$ et al. Role of chemotherapy alone or in combination with hyperthermia in the primary treatment of intraocular retinoblastoma: preliminary results. $\mathrm{Br} J$ Ophthalmol 1998; 82(10): 1154-1158.

9 Lumbroso-Le Rouic L, Aerts I, Lévy-Gabriel C, Dendale R, Sastre $\mathrm{X}$, Esteve $\mathrm{M}$ et al. Conservative treatments of intraocular retinoblastoma. Ophthalmology 2008; 115(8): 1405-1410, 1410.e1401-1402.

10 Lumbroso L, Doz F, Urbieta M, Levy C, Bours D, Asselain B et al. Chemothermotherapy in the management of retinoblastoma. Ophthalmology 2002; 109(6): 1130-1136.

11 Moll A, Imhof S, Meeteren A, Boers M. At what age could screening for familial retinoblastoma be stopped? A register based study 1945-98. Br J Ophthalmol 2000; 84(10): 1170-1172.

12 Abramson D, Mendelsohn M, Servodidio C, Tretter T, Gombos D. Familial retinoblastoma: where and when? Acta Ophthalmol Scand 1998; 76(3): 334-338.

13 Beck M, Balmer A, Dessing C, Pica A, Munier F. First-line chemotherapy with local treatment can prevent externalbeam irradiation and enucleation in low-stage intraocular retinoblastoma. J Clin Oncol 2000; 18(15): 2881-2887.

14 Aerts I, Pacquement H, Doz F, Mosseri V, Desjardins L, Sastre $\mathrm{X}$ et al. Outcome of second malignancies after retinoblastoma: a retrospective analysis of 25 patients treated at the Institut Curie. Eur J Cancer 2004; 40(10): 1522-1529.

15 Egbert P, Donaldson S, Moazed K, Rosenthal A. Visual results and ocular complications following radiotherapy for retinoblastoma. Arch Ophthalmol 1978; 96(10): 1826-1830.

16 Desjardins L, Chefchaouni M, Lumbroso L, Levy C, Asselain B, Bours D et al. Functional results after treatment of retinoblastoma. J AAPOS 2002; 6(2): 108-111.

17 Abramson D, Beaverson K, Chang S, Dunkel I, McCormick B. Outcome following initial external beam radiotherapy in patients with Reese-Ellsworth group $\mathrm{Vb}$ retinoblastoma. Arch Ophthalmol 2004; 122(9): 1316-1323. 